\title{
Neuroimaging of Dementias
}

litions would like to thank Mauricio Castillo, Editor-in-Chief of the American Journal of Neuroradiology (AJNR), for inviting me to be Guest Editor for the AJNR Special Collection, titled Neuroimaging of Dementias. There have been a significant progression of the number of studies and important results in this field. In this collection, more than 250 articles were evaluated, and the ones that were thought to be the most relevant were selected. Selection criteria included articles that 1) were important in the way dementias are diagnosed, 2) had useful imaging techniques, 3) had a high number of citations, 4) were timely, and 5) influenced patient management.

To make these references more accessible, these articles are first grouped by clinical condition, with additional sections that follow dealing with imaging approaches. Articles are first grouped in categories involving general reviews, genetics, types of dementias, and imaging techniques. Because there are overlaps across groups, some articles may be listed under more than one heading.

The general reviews introduce the clinical presentation and imaging patterns associated with dementias. The relation of dementias to pathology is discussed, as well as associations with other disorders. Detailed discussions of the imaging approaches for dementias are included, with references to techniques such as anatomic, functional, and diffusion tensor imaging. A specialized review of neuroimaging of rapidly progressive dementias is discussed in 2 separate parts for easier access, with Part 1 describing neurodegenerative etiologies, and Part 2 discussing prion, inflammatory, neoplastic, and other etiologies. The genetics and clinical presentations of Alzheimer disease are discussed in a separate Genetics Vignette. A companion Genetics Vignette on "Imaging of the 6-OPRI mutation Prion Disease: An Entity Distinct from Typical CJD?" is included. The surprising possibility of changing the structure and size of the cortex is also discussed in an editorial.

The next several groups are based on clinical presentations and discuss imaging findings in normal aging, mild cognitive impairment, Alzheimer disease, and other dementias. This includes studies of changes associated with normal aging, which can be important in the differential diagnosis of cognitive disorders. Studies on mild cognitive impairment have received increased attention recently, because imaging may play a role in the predic-

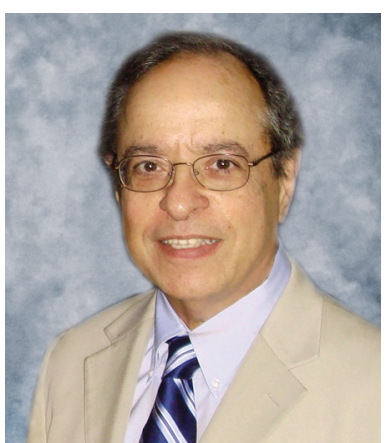

tion of further deterioration and diagnosis. Articles grouped under Alzheimer disease and other dementias describe the utility of neuroimaging in assessing factors such as the effect of aging and conversion to Alzheimer disease, by using various techniques such as PET, SPECT, morphometry, structural findings, and other biomarkers.

The section on anatomy and MR imaging is the longest, which reflects the intense efforts and numerous studies that have been performed to define accurate anatomic measures for dementias. Some of the studies describe the association of dementias with abnormalities of various brain regions, white matter changes, cortical thickness, and other measures. A large study on aging and sex in cognitively healthy elders describes associated brain structural variability. The prediction of subsequent diagnosis of Alzheimer disease atrophy in subjects with mild cognitive impairment is analyzed in a study of temporoparietal MR imaging measures. In terms of other biomarkers, one article describes the role Apolipoprotein $\epsilon 4$, which does not modulate amyloid- $\beta$-associated neurodegeneration in preclinical Alzheimer disease.

The next group, which involves imaging approaches, includes studies that use techniques such as conventional MR imaging, magnetization transfer imaging, voxel-based morphometry, functional imaging, diffusion MR imaging and diffusion tensor imaging, perfusion imaging, magnetoencephalography (MEG), PET and SPECT. Voxel-based morphometry has received increased amounts of attention recently due to its accuracy in defining specific brain regions affected by dementias. Functional imaging has been used to evaluate memory function and more recently to evaluate the connectivity and integrity of resting-state networks in dementias. Diffusion tensor imaging can demonstrate changes in diffusion measures with high sensitivity and specificity for diagnosis. A relevant article on novel white matter tract integrity metrics sensitive to 
Alzheimer disease progression is included. Perfusion imaging and MEG have been found to have some degree of utility, though this has been limited. Molecular imaging techniques such as PET and SPECT are essential because they can track metabolic rates and also target compounds such as amyloid. A study using FDG-PET, SPECT, and structural MR imaging for prediction of rapid conversion to Alzheimer disease in patients with mild cognitive impairment demonstrates the utility of these different approaches.
The selection was in part subjective and I regret that I could not include other important articles that have been published in the AJNR. Nevertheless, this Special Collection should be a useful source of information on the imaging of dementias.

Lucien M. Levy, MD, PhD

Professor of Radiology and Director Neuroradiology George Washington University Medical Center

Washington, DC

http://dx.doi.org/10.3174/ajnr.A3549 